\title{
La grammaire au lycée...qu'en est-il des pratiques de classe ?
}

\section{Abstract:}

In this article, we are interested in the practices of grammatical teaching adopted by the teachers of French as a foreign language. The objective of the analysis of the filmed sessions of teaching is to have on one hand a better understanding of the practices of teaching of the grammar in the secondary school, and on the other hand, to observe if the didactic proposals stemming from the enunciative grammar are applied in class of French.

Keywords: grammar, methods of grammars, grammatical exercises, observation of class.

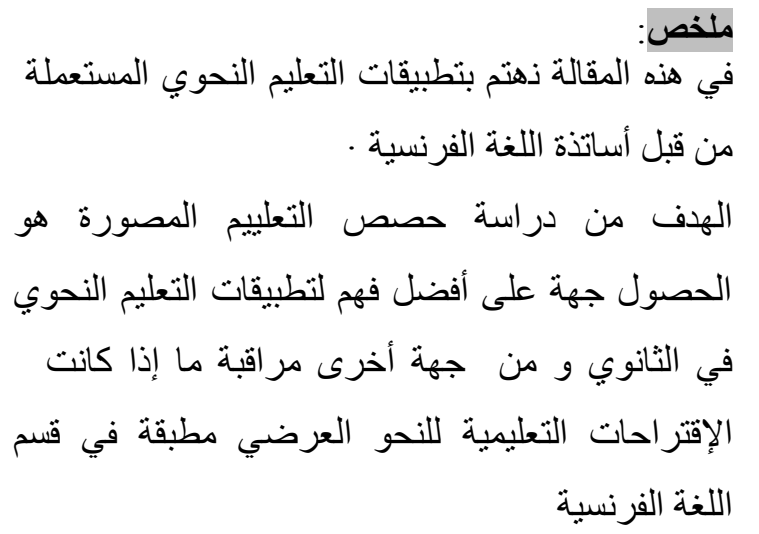

\section{Introduction :}

La présente recherche est née du constat qu'il y a un intérêt de plus en plus important pour l'enseignement des contenus grammaticaux en classe de langue étrangère. Cependant, force est de constater que nous n'assistons pas simplement à un retour de la grammaire en classe de FLE, mais aussi à l'expression presque unanime d'un besoin de renouvellement des pratiques de l'enseignement grammatical. 
Ce changement de vision, du moins en Algérie où le français s'enseigne comme langue étrangère, est dû dans une certaine mesure aux critiques formulées ces dernières années concernant la piètre qualité de la langue parlée et écrite des élèves surtout du niveau secondaire.Ce travail de recherche s'inscrit dans la continuité de notre mémoire de magistère, réalisé sur la problématique de l'enseignement de la grammaire du français en Algérie et dans lequel nous avons expliqué à travers une analyse de productions écrites pourquoi le niveau des élèves du secondaire est en décadence effrénée ces dernières années en particulier dans le domaine de la grammaire.

On ne saurait cependant proposer efficacement des types d'interventions précises sans avoir une description récente de la classe de FLE. Dans cette perspective, il nous est paru essentiel comme point d'appui à notre recherche de connaître, à travers l'analyse de séances d'enseignement filmées, les pratiques d'enseignement grammatical adoptées par les enseignants de français langue étrangère au secondaire et leur perceptions concernant la nature de l'enseignement grammatical : concepts, terminologie, démarches et méthodes utilisées pour la présentation de la grammaire, les exercices grammaticaux.

L'objectif premier de l'analyse des séances d'enseignement filmées est d'avoir une meilleure compréhension des pratiques d'enseignement de la grammaire au secondaire, et d'autre part, d'observer si les propositions didactiques issues de la grammaire énonciative sont appliquées en classe de français. Rappelons que l'examen des programmes et des manuels scolaires du secondaire nous a permis de mettre en évidence la théorie qui sous-tend l'enseignement de la grammaire au lycée à savoir la grammaire énonciative :

«Le présent programme accorde une place plus importante à la

linguistique de l'énonciation qui pose comme préalable qu'il faut

distinguer ce qui est dit (contenu du texte et son contenu informative)

de la présence de l'énonciateur dans son propre discours. Ainsi, tout

discours porte la marque de son énonciateur. En interrogeant ces

traces d'énonciation, en se posant des questions sur le contexte

d'énonciation, les modalités d'inscription du locuteur et de

l'allocutaire dans l'objet texte, le degré d'objectivation du discours,

l'information contenue dans un texte sera appréhendée à un autre niveau de réflexion qui fera prendre conscience au fur et à mesure à

l'apprenant qu'un locuteur est déterminé psychologiquement,

idéologiquement, et culturellement comme il l'est lui-même. »"

(Programme de $2^{\text {ème }}$ A.S : 41).

Les éléments de l'énonciation se manifestent en effet de manière régulière en vue de leur application à l'analyse et l'explication des textes et des points de langue. 
A travers l'analyse des données, nous avons essayé de répondre aux questions suivantes : -Comment sont présentés les contenus grammaticaux ?-Quelles sont les méthodes pédagogiques et les techniques utiliséesdans l'explication des contenus grammaticaux ?-Les concepts utilisés dans l'explication de la grammaire découlent de quelle théorie grammaticale ?-Quelles conclusions pouvons-nous tirer par rapport aux pratiques d'enseignement grammatical de nos sujets?

Démarche méthodologique

Nous avons eu recours à l'observation de classe comme méthode de recueil d'information. Le recours à cet instrument est justifié d'abord par rapport à la nature de la communication, ensuite par rapport à l'étendue de l'accès à l'information. L'observation de classe permet d'établir une communication de plus en plus directe et interactive et un accès à l'information de plus en plus large dans le temps et dans l'espace en comparaison avec d'autres méthodes. Au cours de notre recherche nous avons ciblé deux contextes d'enseignement pour mener des observations sur le terrain, nous avons effectué des séances d'observation avec des classes de première et de deuxième année secondaire lors de la présentation des activités de compréhension de l'écrit (3séances), d'évaluation diagnostique (1séance) et de grammaire (6séances). Nous avons choisi de filmer des séances de compréhension de l'écrit et d'évaluation diagnostique parce que nous avons considéré que les interventions sur l'aspect grammatical peuvent avoir lieu pendant ces séances. Enfin, pour des raisons de confidentialité, nous ne nommerons pas les deux enseignantes volontaires, elles seront désignées par E1 et E2.

Analyse des résultats

\section{-les méthodes et les techniques d'enseignement des contenus} grammaticaux :

En relisant la transcription intégrale des séances auxquelles nous avons assisté nous pouvons constater que les deux enseignantes se rejoignent beaucoup quant à la démarche utilisée dans l'enseignement des contenus grammaticaux, en effet, nous avons remarqué qu'elles utilisent une démarche fondée sur l'exposé et l'explicitation des règles, suivie d'applications conscientes par les élèves, autrement dit, les deux enseignantes observées enseignent la grammaire d'une manière explicite : représentation ou formulation descriptives et explicatives de règles et de fonctionnement de la langue, nous avons également constaté que les deux enseignantes ont opté pour la démarche inductive dans letraitement des notions grammaticales. Par ailleurs, nous avons remarqué que les pratiques reliées à l'enseignement grammatical se font tantôt de façon non planifiée à travers essentiellement les rétroactions correctives :

"E1: il y a une faute là depuis quand chantes-tu /-A : chantes es -/ chantes-tu tu chantes s tu chantes présent de l'indicatif » 
«2 : mis les propos sont mis en cuvres en auvre -/ mis en cuvre entre guillemets ».

Tantôt de façon planifiée à travers :

-le questionnement ciblé :

"E1 : oui nouvelle c'est avec deux $l$, deux l, pourquoi on écrit nouvelle ici avec deux l et pas avec un seul

$A$ : madame star féminin

E1 : oui star est un nom féminin donc nouvelle prend deux $l$ »

-la présentation d'une règle :

$-« E 2$ : ces verbes impersonnels se conjuguent exclusivement à la troisième personne du singulier c'est-à-dire avec le pronom il »

-la mise en évidence d'une règle et l'explication :

"E1: c'est la même chose que l'opposition il y a une petite nuance c'est tout je te dis certes, certes tu as compris la question mais tu n'as pas su répondre c'est-à-dire qu'il y a une partie qui est bonne et une deuxième partie qui n'est pas bonne je te dis, ce n'est pas la même quand je dis il est riche mais il est avare est ce que c'est la même chose que

I- A : non-/ avec certes, certes exprime la concession et le mais exprime l'opposition $》$.

Bien que nous ayons un bon nombre de séquences consacrées à la rétroaction corrective, les enseignantes interviennent ou font intervenir les élèves pour corriger les erreurs, il est évident que les deux enseignantes ont fait beaucoup de travail au niveau des règles de grammaire. Beaucoup d'explications longues sont données. Il semble que les enseignantes expliquent beaucoup en donnant des exemples ou en comparant. Vient ensuite le questionnement ciblé, à savoir une question posée par l'enseignant afin d'attirer l'attention des élèves sur un élément grammatical précis.

Cependant, nous avons noté que pour E1 les interventions portant sur la grammaire se font plutôt de façon non planifiée (rétroactions correctives), beaucoup d'explications brèves sont données, la situation est quelque peu différente pour E2, l'enseignante a davantage eu recours à des techniques planifiées, l'enseignante a fait beaucoup de travail au niveau des règles grammaticales. Elle a par ailleurs travaillé au niveau du questionnement, les explications prennent aussi une grande place.

Les exercices grammaticaux considérés comme des pratiques d'enseignement plus planifiées, sont utilisés en grand nombre dans les classes observées. En effet, dans toutes les séances auxquelles nous avons assisté et qui portent sur l'enseignement grammatical les deux enseignantes achèvent le cours en donnant des exercices d'application. Ces exercices ne sont pas généralement fabriqués par les enseignantes, ils sont repris du manuel de l'élève.

-Métalangage et terminologie 
La lecture de la transcription intégrale de la vidéo, voici ce que nous avons constaté à propos du métalangage et de la terminologie utilisés dans l'explication de la grammaire. Rappelons par ailleurs que l'analyse exhaustive des programmes et des notions grammaticales proposées dans les manuels scolaires du secondaire, nous a permis de mettre en évidence la théorie qui sous-tend l'enseignement de la grammaire au lycée à savoir la linguistique énonciative.

Nous avons remarqué que les points de langue sont souvent mis en relation avec le type de texte étudié, nous avons constaté également que seule la notion grammaticale utile pour l'objectif d'expression visé est étudiée en contexte, il s'agit de la grammaire contextualisée. Ainsi la grammaire se trouve subordonnée à des fins de communication. Les activités proposées sont insérées selon un enchaînement fonctionnel où les leçons de grammaire deviennent complémentaires de toutes les autres.Ainsi,les enseignantes se focalisent sur les notions grammaticales nécessaires à l'acquisition de l'acte d'expression visé. La grammaire paraît au service des compétences de compréhension et de production, nous pouvons citer l'exemple des relations logiques explicites qui sont mises au service du texte argumentatif,en effetE1, est intervenu pour expliquer aux élèves l'utilité de la notions grammaticale dans ce type de texte :

"E1 :...d'accord mais après notre travail on va insister sur la cause et la conséquence et puis je vais vous faire travailler aussi sur l'opposition pourquoi, pourquoi je ne vais pas travailler sur la condition, l'hypothèse pourquoi je ne vais pas prendre le but, $A x:(X x x)$, E1 : parce qu'on est dans le texte /-Ax : argumentatif-/argumentatif et qui dit texte argumentatif dit /-Ax : cause-/ cause argument qui dit argument dit cause c'est clair là c'est pour cela on travaille l'expression de la cause et pour la conséquence pourquoi est ce qu'on travaille la conséquence parce que dans le texte argumentatif on a besoin d'une conclusion et qui dit cause dit conséquence elles fonctionnent ensemble toute cause inversée devient /-Ax : conséquence-/ conséquence c'est clair là c'est pour cela que nous allons travailler en deux les relations de cause et de conséquence, en un on voit les relations logiques explicites en général c'est-à-dire les rapports logiques avec l'exercice 1 et 2 c'est tout et en deux nous allons insister sur la cause conséquence... »

De son côté E2 a montré clairement l'intérêt à étudier la forme impersonnelle dans le texte explicatif, son utilisation permet en effet d'effacer les marques de l'énonciateur et de mettre en valeur les faits. L'emploi des marques explicites de la subjectivité est aussi justifié dans le texte d'anticipation, en effet E2 
explique à ses élèves que dans un texte d'anticipation, considéré comme étant une production subjective en relation directe avec la personnalité, les sentiments et les émotions de la personne qui parle, on y emploi des marques explicites de la subjectivité :

«E2 : Les marques explicites de subjectivité la subjectivité qu'est-ce que la subjectivité comment on peut définir la subjectivité la subjectivité être subjectif c'est exprimer ses idées personnelles, ses points de vue ses sentiments ses émotions ses jugements donc on parle de subjectivité si on a donc une description perso, perso

Ax : personnelle

E2 : personnelle, une description personnelle c'est une description donc subjective on donne nos idées personnelles nos sentiments on donne nos émotions nos jugements etc. d'accord rappeler vous qu'on est dans le texte d'anticipation anticipation c'est l'imagination de ce qui va se produire dans l'avenir donc c'est subjectif l'imagination c'est une description personnelle votre description de l'avenir dépend donc de votre personnalité le texte d'anticipation est un texte subjectif c'est pourquoi on va voir les marques explicites de la subjectivité ».

Nous nous apercevons que la grammaire apparait au service des compétences de compréhension et de production.

En examinant de plus près les choix qui ont été faits par nos deux enseignantes dans l'explication de la grammaire (analyse d'une liste comprenant plus de 100 items utilisés lors des dix séances observées), nous voyons que les deux enseignantes utilisent de manière très large une nomenclature traditionnelle, on trouve en effet le matériel métalinguistique de base comme : verbes, sujet, adjectif, adverbe, préposition, conjonction, féminin, pluriel... Le métalangage utilisé dans l'explication de la grammaire est donc essentiellement celui de la grammaire traditionnelle, les deux enseignantes sont encore très marquées par le paradigme de la grammaire traditionnelle. Elles font encore des analyses qui relèvent de la grammaire traditionnelle et continuent de se raccrocher le plus souvent dans leurs explications aux formes et aux catégories grammaticales traditionnelles. Ces données rejoignent les résultats obtenus par le biais des questionnaires où les participants reconnaissent faire appel aux démarches et au métalangage de la grammaire traditionnelle. Nous avons remarqué, en revanche que les deux enseignantes n'ont pas hésité à utiliser un lexique grammatical spécialisé, celui de la grammaire énonciative, les termes utilisé sont : énoncé, énonciateur, situation d'énonciation, situation de communication, indices de personnes, subjectivité, objectivité, schéma de la communication, discours rapporté..., regardons maintenant de plus près le lexique grammatical utilisé dans chaque séance :

Le discours rapporté : les deux enseignantes semblent être à cheval sur les deux grammaires, en effet, pour expliquer le discours rapporté la structure 
notionnelle de la leçon de E2 a été présentée en deux parties. D'abord elle a présenté aux élèves le discours rapporté en insistant brièvement sur la complexité énonciative qui le caractérise, cette première étape n'a duré que quelques minutes.Ensuite elle a expliqué aux élèves tout au long des deux séances consacrées à la notion qu'il y a deux types de discours rapporté : le discours direct et le discours indirect et que le passage d'un type de discours à l'autre entraine des modifications syntaxiques et morphologiques :

«E2 : donc on remplace la conjonction de subordination que par les deux points et les guillemets les deux points qui annoncent donc le début le commencement des du discours direct et les guillemets qui encadrent le discours direct allez quoi d'autre allez les autres modifications allez. A : les pronoms personnels

E2 : oui les pronoms personnels et les possessifs aussi. A : madame les indicateurs de lieu

E2 : oui les indicateurs de lieu ou cc de lieu. A : les indicateurs de temps

E2 : les indicateurs de lieu ou cc de temps. A : le temps du verbe

E2 : le temps du verbe. $A$ : le mode du verbe

E2 : oui le temps et le mode du verbe, »

Le métalangage utilisé relève plutôt de la grammaire traditionnelle. Au niveau des exercices proposés, on a recours à la grammaire transformationnelle à travers des exercices de transformation où les élèves doivent transformer des passages du style indirect au style direct. La notion de discours rapporté traditionnellement traitée en grammaire semble relativement peu affectée par la perspective énonciative récemment introduite dans les programmes et les manuels : on insiste sur les transformations syntaxiques et morphologiques auxquelles donne lieu le passage d'un discours à l'autre mais on ne se place pas dans la perspective des reformulations que peut opérer l'énonciateur de son discours ni de ce qu'implique pour lui l'utilisation des différents styles.

La forme impersonnelle : nous avons remarqué que la même démarche a été employée dans le traitement de la forme impersonnelle, E2 a d'abord expliqué aux élèves, en utilisant une terminologie simple, que la forme impersonnelle peut être considérée comme un marqueur linguistique de modalité, elle permet d'exprimer une certaine attitude du sujet énonciateur par rapport au contenu propositionnel de son énoncé, voici son explication :

«E2 : alors on vous dit que l'emploi de la forme impersonnelle dans le texte explicatif permet d'effacer la présence de l'énonciateur, l'énonciateur c'est la personne, eh c'est la personne qui

$A: l^{\prime}$ auteur

E2 : oui c'est l'auteur et donc c'est la personne qui parle c'est la personne qui explique donc l'énonciateur c'est celui qui explique dans un texte explicatif, c'est l'auteur d'un texte explicatif donc on utilise les 
tournures impersonnelle pour effacer la présence de celui qui explique dans un texte explicatif de l'énonciateur d'accord parce que dans un texte explicatif on insiste sur les faits scientifiques on n'insiste pas sur la personne qui parle ».

La forme impersonnelle permet donc de marquer une certaine distance entre le sujet énonciateur et son discours, cette première phase de la leçon n'a duré que quelques minutes pendant lesquelles l'enseignante a utilisé les termes relevant de la linguistique énonciative.Le reste de la séance a été consacré à l'explication de la structure syntaxique d'une phrase à la forme impersonnelle, l'enseignante a fait appel à la grammaire transformationnelle à travers deux exercices de transformation, là aussi, on insiste sur le passage d'une forme à une autre mais on ne se place pas dans la perspective des reformulations et des choix qui peuvent être opérés par l'énonciateur.

Les relations logiques explicites : lors de la séance qui porte sur les relations logiques explicites, E1, a soulevé la fonction discursive de certaines unités linguistiques dans l'organisation générale du discours en expliquant à ses élèves que la fonction de ces unités consiste d'une part à marquer les différentes parties du discours et d'autre part, à expliciter les liens logiques ou argumentatifs qui existent entre les énoncés :

" E1 : bon alors ce qu'il faut retenir c'est que pour avoir un sens il faut qu'on utilise des connecteurs des mots qui sont porteurs sens alors on vous dit dans un énoncé les éléments porteurs de sens informations essentielles illustrations sont disposés de façon à exprimer un rapport logique...»

«E1 : parce qu'on est dans le texte /-Ax: argumentatif-/argumentatif et qui dit texte argumentatif dit /-Ax: cause-/ cause argument qui dit argument dit cause c'est clair là c'est pour cela on travaille l'expression de la cause et pour la conséquence pourquoi est ce qu'on travaille la conséquence parce que dans le texte argumentatif on a besoin d'une conclusion et qui dit cause dit conséquence elles fonctionnent ensemble toute cause inversée devient /-Ax : conséquence-/ 》.

Les marques explicites de subjectivité :nous avons remarqué que la structure notionnelle de la leçon a été présentée en deux étapes. L'enseignante a consacré une première étape de la leçon pour faire la distinction entre un texte subjectif et un texte objectif, pour ce faire elle a mis en évidence la fonction de modalisation de certaines unités langagières qui permettent au locuteur d'exprimer son point de vue, ses sentiments, ses émotions, ses idées, ses certitudes, ses jugements, bref, sa manière d'envisager les contenus relatés :

«E2 : une description personnelle c'est une description donc subjective on donne nos idées personnelles nos sentiments on donne nos émotions nos jugements etc. d'accord rappeler vous qu'on est dans le texte 
d'anticipation anticipation c'est l'imagination de ce qui va se produire dans l'avenir donc c'est subjectif l'imagination c'est une description personnelle votre description de l'avenir dépend donc de votre personnalité le texte d'anticipation est un texte subjectif...

E2 : la description personnelle subjective elle est en rapport avec celui qui parle donc celui qui produit l'énoncé l'auteur de l'énoncé d'accord donc subjectif c'est le contraire de Ax : objectif "

Dans une deuxième étape de la leçon, E2 a expliqué à ses élèves que ce phénomène est souvent assuré par des verbes, des adjectifs mais aussi par des adverbes et des locutions adverbiales (terminologie traditionnelle), elle a proposé enfin une longue liste de quelques marques de la subjectivité qui comprend essentiellement des verbes de sentiment, des verbes d'opinion, des verbes de perception, des verbes de jugement.L'enseignante a opté pour une approche taxinomique, elle a donné une liste d'indices mais peu de principes de fonctionnement, cette démarche n'aide guère les élèves à résoudre leurs difficultés à inscrire leur subjectivité dans leursproductions. Il ne s'agit pas de négliger l'analyse des marques de subjectivité indispensable à l'enrichissement du bagage linguistique des élèves mais de procéder à une démarche didactique autorisant une sélection réfléchie de ces outils par les élèves.

La subordonnée relative: Contrairement à ce que nous avons constaté en analysant les séances précédentes, nous avons remarqué que E2 a employé essentiellement une terminologie grammaticale traditionnelle dans le traitement de la subordonnée relative. Voici son explication :

«E2 : la subordonnée relative est une subordonnée introduite oui subordonnée introduite par un pronom relatif, elle apporte généralement une précision sur le mot qui la précède, elle apporte j'ai dit généralement une précision, une précision sur le mot sur le mot qui la précède / elle chut elle est supprimable, oui elle est donc supprimable //le pronom relatif représente un mot de la principale c'est son / c'est son antécédent // tu prends maintenant les deux tableaux le premier tableau vous avez les pronoms relatifs simples et le deuxième tableau vous avez les pronoms relatifs, les pronoms relatifs on a les simples et les

Ax : les composés 》

Enfin nous pouvons dire que les deux enseignantes utilisent un métalangage composite, beaucoup de termes utilisés sont en effet issus de la grammaire traditionnelle, d'autres sont issus de la grammaire énonciative. Ces résultats rejoignent ceux obtenus à partir des déclarations des enseignants qui affirment utiliser dans leurs cours un mélange de grammaire traditionnelle et de grammaire énonciative. Nous pouvons dire enfin qu'en dépit de l'emploi occasionnel de termes de la grammaire énonciative ce qui est fait relève 
essentiellement de la grammaire traditionnelle. En effet, Malgré le recours à des moyens d'enseignement de la grammaire énonciative, on sent l'esprit de la grammaire traditionnelle chez les deux enseignantes en raison d'une part du caractère magistral de leurs interventions, et d'autre part du lexique grammatical utilisé. Nous avons constaté également que les références aux notions développées dans le cadre des théories de l'énonciation se font de plus en plus fréquentes. Cependant les enseignantes n'organisent pas une présentation cohérente et raisonnée des outils et des notions conçus à partir de cette théorie. Les données de l'énonciation se manifestent régulièrement en vue de leur application à l'explication des textes ou des points de langue, les notions de relations logiques explicites, de discours rapporté, de forme impersonnelle et des marques explicites de la subjectivité sont mises explicitement en perspective avec la notion d'énonciation à travers une terminologie simplifiée. Vulgarisée en classe de français, nous avons remarqué que l'énonciation ne fait généralement pas l'objet d'une définition en tant que telle, elle est employée souvent dans l'explication des textes et des points de langue, elle est réduite à une simple analyse de certaines notions grammaticales.

\section{Conclusion}

Tout au long de cette recherche, nous avons visé deux objectifs. En premier lieu, il s'agissait d'avoir une meilleure compréhension des pratiques d'enseignement de la grammaire au secondaire. En deuxième lieu, il s'agissait d'observer si les propositions linguistiques issues la grammaire énonciative (méthodes et notions) étaient appliquées en salle de classe.

Les résultats présentés semblent indiquer que l'enseignement grammatical est bel et bien présent dans les classes de français. Les observations en classe ont mis en lumière que les enseignants choisis dans le cadre de cette étude font usage de techniques d'enseignement grammatical, d'exercices grammaticaux et d'une terminologie grammatical.Voici quelques constats d'ordre linguistique et pédagogique :

-Les notions grammaticales étudiées sont souvent mises en relation avec le type de texte étudié,

-Les enseignantes utilisent une démarche explicite fondée sur l'exposé et l'explication des règles grammaticales et optent pour la démarche inductive.Les interventions portant sur la grammaire se font tantôt de façon non planifiée (rétroaction corrective), tantôt de façon planifiée (questionnement ciblé, explication, présentation et mise en évidence d'une rège).Les exercices grammaticaux considérés comme des pratiques d'enseignement plus planifiées sont utilisés en grand nombre.

-La grammaire apparait au service des compétences de compréhension et de production.

-Les deux enseignantes utilisent de manière très large une nomenclature traditionnelle, le métalangage utilisé dans l'explication de la grammaire est 
essentiellement celui de la grammaire traditionnelle. Les enseignantes utilisent un métalangage composite : beaucoup de termes utilisés sont issus de la grammaire traditionnelle, d'autres sont issus de la grammaire énonciative. Les références aux notions développées dans le cadre des théories de l'énonciation se font de plus en plus fréquentes.

-L'énonciation est réduite à une analyse limitée à quelques notions du langage comme le discours rapporté, la forme impersonnelle, la modalisation. Ces notions sont mises en perspective avec l'énonciation.

On ne saurait certes tirer des conclusions générales de cette enquête qui sont loin de représenter toutes les situations d'enseignement/apprentissage du français, mais elle met en évidence un certain nombre d'éléments importants.

Bibliographie :

1) Authier-Revuz, J. (1993) : "Repères dans le champ du discours rapporté", in l'information grammaticale, $n^{\circ} 56$.

2) Besse, H., (1995) : Méthodes et pratiques des manuels de langue, Didier, Paris.

3) Besse, H., Porquier, R. (1984): Grammaire et didactique des langues, Hatier, Paris.

4) Boch, F. et Grossmann, F., (2009): "Polyphonie linguistique : modalisation et discours rapporté dans les manuels scolaires de français", SCRIPTA BeloHorizante, V. 13, $\mathrm{n}^{\circ} 24,1^{\mathrm{e}} \mathrm{sem}$.

5) Chartrand, S.-G. (1995) : "Pour un nouvel enseignement de la grammaire". Collectif sous la direction de S.-G. Chartrand, Montréal, Les Éditions Logiques, 417 pages.

6) Cuq, J.P. (1996) : Une introduction à la didactique de la grammaire en français langue étrangère, Les Éditions Didier, Paris.

7) Cuq, J.-P. (2002) : "Du discours du linguiste au discours pédagogique : tribulations de quelques concepts grammaticaux", revue de didactologie des langues- cultures $1, \mathrm{~N}^{\circ} 125, \mathrm{p} .83-95$

8) Cuq, J.-P., et L. Gruca. (2002) : Cours de didactique du français langue étrangère et seconde. Grenoble : Presses universitaires de Grenoble.

9) Simard, D., (2001) : "Observation des techniques de mise en évidence de l'input utilisées par les enseignants d'anglais L2 du primaire". Université du Québec à Trois-Rivières.

10) Trévise A. (1993) : "Acquisition/ apprentissage/ enseignement d'une L2 : modes d'observation, modes d'intervention". Etudes de linguistique appliquée, 92, 38-50. 